\title{
Factores de éxito de los MOOC: algunas consideraciones críticas
}

\author{
Raquel Poy ${ }^{1}$, Audilio Gonzales-Aguilar ${ }^{2}$ \\ rpoyc@unileon.es, audilio.gonzales@univ-montp3.fr
}

1 Departamento de Didáctica General, Específica y Teoría de la Educación, Universidad de León, Campus de Vegazana, s/n, 24071, León, España.

2 Departamento de Documentación, Universidad Paul Valéry Montpellier 3, Route de Mende, 34199 Montpellier Cedex 5, Francia

DOI: 10.4304/risti.e1.105-118

\begin{abstract}
Resumen: Los cursos online abiertos y masivos (MOOC), una de las herramientas que ha surgido de la combinación de e-learning y modelos de redes sociales, se ha integrado en muchos programas educativos transformando las organizaciones e-learning a nivel mundial. En cuanto tecnología de aprendizaje relativamente nueva, se identifican los factores determinantes de su éxito a partir de la revisión de la literatura científica y de las estadísticas propias de las principales plataformas. A partir de los estudios previos, los cuatro factores críticos son el diseño de software educativo, las tasas de abandono, el alcance de la universalización, y la estrategia de negocio subyacente a esta oferta educativa. Se utilizó una muestra de 35 plataformas con más de 7.000 cursos para revisar los factores de análisis propuestos. Las áreas que parecen ser más críticas son la baja interactividad y un modelo de negocio diseñado sin la adecuada participación de la comunidad educativa.
\end{abstract}

Palabras-clave: Cursos COMA, teleformación, brecha digital, abandono educacional, software educativo.

\section{MOOC success factors: some critical considerations}

\begin{abstract}
Massive online open courses (MOOC) are reshaping e-learning organizations worldwide. E-learning strategists have begun increasingly to utilize and integrate MOOC platforms in their online learning systems. MOOC, one of the tools that have emerged from the combination of e-learning courses and social networks models, has been integrated into many educational programmes. Since MOOC are a relatively new learning technology, this paper is intended to identify and measure its critical success factors from the review of scientific literature and the statistics shown for major platforms. In line with the literature, four factors were identified and measured, namely, educational software design, dropout rates, universal scope, and business strategy. A sample of 35 MOOC platforms with more than 7000 courses was used to review the proposed analytical factors. The two
\end{abstract}


areas that appear to be most critical are low interactivity and a business strategy designed without adequate participation of the educational community.

Keywords: Massive online open courses MOOC, e-learning, digital divide, educational dropping-out, educational software.

\section{Introducción}

Los cursos en formato Mooc, cuyas siglas en ingles responden al concepto de Massive Open Online Courses, o Cursos Abiertos de Acceso Masivo, son un desarrollo reciente en el ámbito de la teleformación o formación a distancia, que permite a través de foros participativos la realización de actividades formativas en las que el grado de involucración de educadores y educandos es mayor que los tradicionales productos de elearning como vídeos, lecturas o ejercicios prácticos.

Para algunos analistas, las herramientas Web 2.0 que subyacen a estas nuevas formas de e-learning ocasionan una transformación del espacio social al ofrecer a los individuos nuevas oportunidades de aprendizaje rompiendo las barreras tradicionales del espacio y el tiempo de la formación reglada en el modelo global de e-learning y que se basaba en estar estrechamente monitorizada por el profesor o tutor (Brown y Adler, 2008; Kop, 2011; Esposito, 2012).

$\mathrm{El}$ auge de las plataformas Web que ofrecen este tipo de cursos ha llevado a algunos a señalar que 2012 fue el año de las MOOC e incluso que se comenzaba a vislumbrar la esperanzadora era del MOOC 3.0 ${ }^{1}$. Sin embargo, esos mismos analistas, apenas un año después, comienzan a detectar que no todo transcurre tan deprisa como parecía.

Y es que, en apenas cinco años, el vertiginoso ascenso de las Plataformas MOOC ha llevado a centros e instituciones educativas de todo el mundo a disponer de una estrategia propia (Kohler et. al, 2013), cuando no de una plataforma con menor o mayor nivel de desarrollo ${ }^{2}$. Sin embargo, a la luz de los actuales estudios sobre este fenómeno, cabe preguntarse si se está produciendo el auge y a la vez el punto de inflexión en este crecimiento vertiginoso. ¿Estamos asistiendo a una crisis en el modelo MOOC? ¿Hasta qué punto es una crisis que obedece a cambios en el contexto y el mercado educativo o, por el contrario, obedece a errores en la estrategia y diseño de estos productos de e-learning especialmente diseñados para entornos Web?

En el presente articulo analizamos, a partir de un muestreo exhaustivo de las principales iniciativas de Plataformas MOOC, si existen en este punto posibles planteamientos que estén dificultando el éxito de los MOOC.

${ }^{1}$ http://www.ecampusnews.com/around-the-web/american-higher-education-and-the-Mooc-3o-era/.

2 Es el caso en particular de Courlis http://courlis-pf.univ-lorraine.fr/ (cursos de estadística aplicada) 


\section{Análisis de las plataformas MOOC}

Las plataformas que a comienzos de 2013 se han constituido en diferentes puntos del globo incluyen diversos tipos de software, diseño y funcionalidades, pero en su conjunto todas comparten una serie de características comunes. Hemos seleccionado el conjunto de plataformas MOOC más conocidas, incluyendo una serie de plataformas españolas, a efectos de reflejar los volúmenes de cursos y alumnos que han alcanzado hasta la fecha.

Tabla 1 - Principales Plataformas MOOC a nivel internacional (elaboración propia a partir de muestreo de fecha Julio 2013)

\begin{tabular}{|c|c|c|c|}
\hline Plataforma & URL & $\begin{array}{l}\text { Número } \\
\text { de cursos }\end{array}$ & País \\
\hline Coursera & https://www.coursera.org/ & 416 & USA \\
\hline EdX & https://www.edx.org/ & 56 & USA \\
\hline Udemy & http://www.udemy.com/ & & USA \\
\hline Udacity & http://www.udacity.com/ & 30 & USA \\
\hline $\begin{array}{l}\text { OpenClass-BETA } \\
\text { de Pearson Ltd. }\end{array}$ & http://www.openclass.com/open/home/index & -- & UK \\
\hline Lore (Noodle) & http://lore.com/ & -- & USA \\
\hline Canvas & https://www.canvas.net/ & -- & USA \\
\hline Venturelab & http://venturelab.stanford.edu/ & 12 & USA \\
\hline Coursesites & https://www.coursesites.com & 32 & USA \\
\hline OpenCourseWare & http://www.ocwconsortium.org/ & 3.500 & USA \\
\hline $\mathrm{P} 2 \mathrm{PU}$ & https://p2pu.org/es/ & -- & USA \\
\hline $\begin{array}{l}\text { Google Course } \\
\text { Builder }\end{array}$ & http://code.google.com/p/course-builder/ & 40 & USA \\
\hline $\begin{array}{l}\text { OpenLearn } \\
\text { LabSpace }\end{array}$ & $\begin{array}{l}\text { http://www.open.edu/openlearn/ } \\
\text { http://labspace.open.ac.uk/ }\end{array}$ & $>1000$ & UK \\
\hline $\begin{array}{l}\text { Open Learning } \\
\text { Initiative- } \\
\text { Carnegie Mellon } \\
\text { Univ. }\end{array}$ & http://oli.cmu.edu/ & 18 & USA \\
\hline $\begin{array}{l}\text { Leuphana Digital } \\
\text { School }\end{array}$ & http://digital.leuphana.de/ & 1 & GER \\
\hline Knight Center & http://knightcenter.utexas.edu/distancelearning & 3 & USA \\
\hline OpenHPI & https://openhpi.de/ & 2 & GER \\
\hline MRUniversity & http://mruniversity.com/ & 7 & USA \\
\hline OpenLearning & https://www.openlearning.com/ & 32 & AUS \\
\hline
\end{tabular}




\begin{tabular}{|c|c|c|c|}
\hline Plataforma & URL & $\begin{array}{l}\text { Número } \\
\text { de cursos }\end{array}$ & País \\
\hline ALISON & http://alison.com/ & 550 & USA \\
\hline $\begin{array}{l}\text { University of the } \\
\text { people }\end{array}$ & http://www.uopeople.org/ & 75 & USA \\
\hline Saylor.org & http://www.saylor.org/ & 278 & USA \\
\hline $\begin{array}{l}\text { Symynd (Share } \\
\text { your mind) }\end{array}$ & http://www.symynd.com/ & 9 & USA \\
\hline $\begin{array}{l}\text { Open Yale } \\
\text { Courses }\end{array}$ & http://oyc.yale.edu/ & 50 & USA \\
\hline GCF Learn Free & http://www.gcflearnfree.org/ & 750 & USA \\
\hline Nixty & http://nixty.com/ & $>200$ & USA \\
\hline SantaFe MOOCs & http://www.santafe.edu/Mooc/subscribe & 1 & USA \\
\hline Unx & http://www.redunx.org/web/guest/home & 5 & ES \\
\hline UnedComa & http://unedcoma.es/ & 20 & ES \\
\hline Crypt4you & http://www.criptored.upm.es/crypt4you/portada.html & 2 & ES \\
\hline MiriadaX & http://miriadax.net/ & 100 & ES \\
\hline $\begin{array}{l}\text { UPVX- } \\
\text { Universidad } \\
\text { Politécnica de } \\
\text { Valencia }\end{array}$ & http://www.upvx.es/ & 13 & ES \\
\hline $\begin{array}{l}\text { Bureau Veritas } \\
\text { Business School } \\
\text { MOOC-España }\end{array}$ & $\begin{array}{l}\text { http://www.bvbusiness- } \\
\text { school.com/cursoselearning/cursos-Mooc-abiertos- } \\
\text { online.aspx }\end{array}$ & 1 & ES \\
\hline
\end{tabular}

Expresamente excluimos de este muestreo las plataformas Academia Herat, Open Michigan y Flexilearn, ya que podrían no tener la consideración completa de tratarse de un MOOC, dado que no permiten la gestión integral de los cursos que albergan, o Webcast Berkeley, que únicamente contiene vídeos educativos3.

También encontramos Plataformas especializadas en la formación en lenguajes de programación como son Codeacademy o CodeSchool 4 , muy específicas para colectivos de programadores, que merecen ser mencionadas, así como existen casos de Plataformas no abiertas o de pago, como el caso de las desarrolladas por la Minerva University 5 o la privada eLearning Center de Texas, aunque es un modelo de negocio el de cursos de pago que ha elegido Plataformas de amplio alcance como Udemy, que combina una oferta gratuita con otra de pago.

3 Cfr. http://www.academicearth.org/; https://open.umich.edu/;

http://www.ignouflexilearn.ac.in/flexilearn/; http://www.futurelearn.com/;

http://webcast.berkeley.edu.

${ }^{4}$ Cfr. http://www.codecademy.com/; http://www.codeschool.com/.

5 Cfr. http://www.minervaproject.com/. 
En Europa se está promoviendo la formación de consorcios que permitan disponer de plataformas multiinstitucionales que sean una alternativa a las estadounidenses, destacando la plataforma liderada por Telefónica S. A. y el Banco de Santander en España (Miriadax), con más de un centenar de cursos procedentes de dos decenas de universidades, o en el caso de Reino Unido, la plataforma FutureLearn que nace asimismo como alternativa a las plataformas americanas, aunque todavía no ha abierto oficialmente, y a partir de un consorcio formado por 12 universidades británicas que se han comprometido a crear cursos para dicha plataforma ${ }^{6}$.

Volviendo a nuestro análisis, podemos extraer una serie de impresiones iniciales tras realizar un examen detallado sobre la información ofrecida por las Plataformas en sus Webs.

Como consideraciones generales sobre las MOOC, en primer lugar cabe destacar la escasa transparencia en sus cifras estadísticas, aunque por motivos lógicos la visibilidad del número de cursos ofertados en sus catálogos es fácil de recoger, en ocasiones el formato de curso no es estándar ni comparable, ya que en muchos casos denominan "curso" a sencillas lecciones que apenas tienen 3-5 minutos de duración y que no requieren un seguimiento académico mínimo, como sucede en el caso de la plataforma de The Open University.

En segundo lugar, cuando alguna plataforma revela el número de alumnos adquiridos o acumulados, no distingue cuáles han mantenido una escolarización completa en su plataforma o si se trata de usuarios contados en repetidas ocasiones como distintas personas. Tampoco aparecen datos sobre tasas de éxito o abandono educativo, pero en algunos casos se publicitan o recogen en estudios ad hoc, lo que confirma unas tasas de abandono que por término medio oscilan entre el 75 y el 95\% de la mayoría de los cursos, como referiremos a continuación:

- Elevadas tasas de abandono. En general, las tasas de abandono confesas oscilan entre el 75 y el 90\% de media. Esta estimación podría ser realista habida cuenta de que en ocasiones se cuenta como estudiante que ha superado el curso aquel que no ha tenido que superar un control por un tercero para comprobar que efectivamente ha conseguido alcanzar los objetivos del mismo. Los primeros estudios de usuario apuntan a que las elevadas tasas de abandono pueden indicar un error en el diseño centrado en el usuario.

El estudio en profundidad del primer curso de la célebre plataforma edX, desarrollada por el consorcio liderado por el MIT y la Universidad de Harvard, y que reunió la abrumadora cifra de $\mathbf{1 5 5 . 0 0 0}$ estudiantes entre Marzo y Junio de 2012 (Breslow, Pritchard, DeBoer, Stump, Ho \& Seaton, 2013), apunta que apenas un 10\% de los alumnos superó el curso, y sólo un 3\% participó en el Foro de Debate abierto en el curso. En cuanto a los alumnos que obtuvieron el certificado de superación del curso, su participación en el Foro sí habría superado el 53\%.

${ }^{6} \mathrm{Cfr}$.

http://www.timeshighereducation.co.uk/story.asp? sectioncode $=26 \&$ storycode $=422137 \& \mathrm{c}=1$ 
- Incertidumbre en el modelo de negocio MOOC. Existe en los MOOC un modelo económico y estratégico sostenido por la innovación y el marketing interactivo (Derycke, A., 2013). Las Plataformas no tienen definido un modelo de negocio contrastado y se ubican entre la estrategia publicitaria de edX y la de posicionamiento en un mercado abonado todavía al capital riesgo. Coursera ha recaudado hasta 65 millones de dólares de fondos de capital riesgo para su consolidación en el mercado universitario durante 2013, y Canvas de Instructure ha seguido el mismo camino con una ampliación hasta 50 millones de dólares en este su quinto año de rodadura. Los niveles de retorno económico declarados por los promotores no parecen apuntar el optimismo. Sin duda el referente principal de este conjunto de Plataformas MOOC es la entidad sin ánimo de lucro constituida por Harvard y el MIT, el conocido edX, cuyos principios declarados son expandir la educación superior a nivel global para cualquier estudiante, publicitando el elevado nivel de calidad de los educadores de sus instituciones, incluidos sus partners de la Universidad de California-Berkeley y la Universidad de Texas7, bajo el slogan "los mejores profesores y universidades", e incluyendo una curiosa llamada a la capacidad para realizar amistades a través de sus redes participativas.

Quizás la supuesta "amenaza" al método tradicional de enseñanza universitaria que representarían los MOOC no sea tal debido al subdesarrollo de la estrategia institucional que están empleando las universidades a la hora de promover estos productos. En este sentido, Teplechuk (2013) cuestiona la estrategia corporativa en cuanto el modelo de negocio que subyace a este tipo de cursos gratuitos y abiertos sólo es sostenible mediante un esfuerzo por conseguir retornos económicos secundarios (p. e. tasas por expedición de certificados de superación del curso), lo que con una tasa media de alumnos que superan los cursos MOOC que apenas supera el 12\%, en este caso en la plataforma de la Universidad de Edinburgo, los convierte en notablemente deficitarios (Teplechuk, 2013: 37-38).

- Objetivo de universalización no alcanzado. En tercer lugar, el pretendido objetivo de democratizar y extender gratuitamente a nivel global y masivo la educación superior gracias a la tecnología, podría estar fracasando a tenor de los resultados sobre el alcance de los cursos MOOC entre el público objetivo. Los estudios que proliferan sobre el fenómeno MOOC apuntan a que el acceso masivo está siendo desigual en función de los perfiles que los alumnos de este tipo de plataformas exhiben, insinuando que la brecha digital se reproduce bajo nuevas formas ante estas plataformas, y resultando beneficiados la elitista comunidad académica y profesional, conjuntamente con los usuarios avanzados de nuevas tecnologías y pertenecientes mayoritariamente a países desarrollados.

- ¿Errores en el diseño del producto software? Una hipótesis que hemos barajado en nuestro análisis es la relativa a posibles errores de estrategia y diseño del software MOOC desde la perspectiva de usuario, para lo cual hemos

\footnotetext{
7 También la Universidad de Stanford ha renunciado a su plataforma MOOC Class2go para integrarse desde Junio de 2013 como partner de la plataforma edX, aunque no como parte del consorcio, sino asociado a una plataforma de software abierto que considera más adecuada a sus intereses.
} 
comprobado la adecuación a los modelos pedagógicos más generalizados en el ámbito del e-learning.

\section{Diseño del software educativo MOOC}

El diseño pedagógico de las plataformas MOOC, es sin duda uno de los elementos más relevantes de cara a optimizar los resultados del aprendizaje de los usuarios de estas plataformas, dado que para que sea posible la puesta en marcha de los procesos educativos, es necesario que se tenga en cuenta, en primer lugar, la fundamentación teórica del modelo pedagógico que los sustenta a fin de determinar las necesidades pedagógicas a las que se les ha de dar respuesta cuando se procede al diseño pedagógico de los entornos virtuales de aprendizaje, cuyo rasgo propio es que el interfaz entre el sujeto que aprende y el diseñador de la práctica educativa es la tecnología (García, 2002).

Tanto las plataformas MOOC como otros productos elearning denominados como software educativo 3.0. y que incluye, por ejemplo los denominados juegos serios (serious games) procedentes del Digital Gamebased Learning, responden a la necesidad de emplear metodologías innovadoras para la consecución de una formación de impacto en el ámbito de los procesos de enseñanza aprendizaje en entornos virtuales de aprendizaje, principalmente en cuanto al grado de participación activa proporcionado al sujeto del aprendizaje (Prensky, 2010).

Precisamente los entornos virtuales de aprendizaje tienen cada vez mayor presencia en el conjunto de la oferta formativa, pudiendo afirmar que, en general, la población que utiliza los actuales canales de aprendizaje on line manifiesta nuevas tendencias como el hecho de tener una mayor demanda de contenidos innovadores respecto a los más tradicionales modelos de aprendizaje propios de la enseñanza presencial (Krichen, 2007). En consecuencia en ambos casos, las plataformas MOOC responden a esa nueva demanda de contenidos innovadores para las nuevas generaciones de usuarios crecientemente inmersos en la sociedad del conocimiento y de la información.

Con el fin de analizar desde el punto de vista del diseño pedagógico, la construcción de las diferentes plataformas MOOC, hemos utilizado el modelo de Freitas y Jarvis (2007) que toma en cuenta tres elementos de análisis más la representación: el contexto, el aprendiz, y la pedagogía usada, además de la propia representación digital del producto e-learning, como cuarto elemento adicional.

El diseño pedagógico de las plataformas MOOC es necesario que tenga en cuenta :

- En primer lugar, la fundamentación teórica del modelo pedagógico que los sustenta a fin de determinar las necesidades pedagógicas a las que se les ha de dar respuesta cuando se procede al diseño pedagógico de los entornos virtuales de aprendizaje, cuyo rasgo propio es que el interfaz entre el sujeto que aprende y el diseñador de la práctica educativa es la tecnología (García, 2002).

- En segundo lugar, tanto las plataformas MOOC como otros productos elearning software educativo 3.o. que incluyen por ejemplo los denominados juegos serios o Digital Gamebased Learning) - responden a la necesidad de emplear metodologías innovadoras para la consecución de una formación de impacto en el 
ámbito de los procesos de enseñanza aprendizaje en entornos virtuales de aprendizaje, principalmente en cuanto al grado de participación activa proporcionado al sujeto del aprendizaje (Prensky, 2010).

En consecuencia en ambos casos, las plataformas MOOC responden a esa nueva demanda de contenidos innovadores para las nuevas generaciones de usuarios crecientemente inmersos en la sociedad del conocimiento y de la información (Krichen, 2007).

\subsection{Plataformas interactivas}

Son precisamente estas herramientas de participación interactiva derivadas de la Web 2.0 las que convierten a las Plataformas MOOC en instrumentos de aprendizaje ambiental o situacional (McLellan, 1997; Dziorny, 2007). Este entorno o contexto en el que se produce el aprendizaje, se basa en proporcionar recursos de apoyo a los que se tiene acceso durante el proceso de aprendizaje, y que por definición se basa en un acceso abierto y regido por el principio de flexibilidad en cuánto al cómo y cuándo accede el estudiante. Asimismo, en las plataformas MOOC el ambiente de aprendizaje es virtual, en cuanto los aprendizajes no se llevan a cabo en un lugar concreto y se reproduce virtualmente un aula o espacio que reúne las condiciones para optimizar el proceso de aprendizaje favoreciendo la adquisición de contenidos, experiencias y procesos pedagógicos, a partir de actividades dialógicas que facilitan intercambios de conocimientos (Avila y Bosco, 2001).

Sin embargo, el nivel de participación que parecen mostrar los usuarios de los cursos MOOC aparenta distar mucho del propósito que anima su diseño interactivo. Un estudio en curso realizado por Katy Jordan, de la Universidad de Texas, para un total de 29 MOOCs, advirtió que la tasa de éxito era de sólo el 6,8\% y cinco de los seis cursos con mayor tasa de éxito no tenían ningún tipo de control o evaluación de tercera persona sobre el nivel de adquisición de conocimientos o habilidades por parte del alumno, por lo que la tasa real podría ser menor (Parr, 2013). Otros estudios de caso coinciden en tasas medias de abandono entre el 80 y el 95\% (Cross, 2013).

Por su parte, el estudio en profundidad del primer curso de la célebre plataforma edX, desarrollada por el consorcio liderado por el MIT y la Universidad de Harvard, y que reunió la abrumadora cifra de 155.00o estudiantes entre Marzo y Junio de 2012 (Breslow, Pritchard, DeBoer, Stump, Ho \& Seaton, 2013), apunta que apenas un 10\% de los alumnos superó el curso, y sólo un 3\% participó en el Foro de Debate abierto en el curso. En cuanto a los alumnos que obtuvieron el certificado de superación del curso, su participación en el Foro sí habría superado el 53\%.

Un estudio desarrollado dirigido por las empresa Qualtrics y Instructure, proveedores de la plataforma Canvas y de 425 centros superiores estadounidenses en su entorno MOOC, y que ha sido publicado en julio de 20138 señala que el principal atractivo de los cursos MOOC residiría en particular en su carácter gratuito, con un $75 \%$ de respuestas que priorizan este elemento como el factor primario para realizarlos.

8 Cfr. https://www.instructure.com/press-releases/qualtrics-and-instructure-reveal-Moocstudents-top-motivations. 
En cualquier caso sí parece claro que el grado de manejo en habilidades digitales (digital skills) resulta un predictor de la accesibilidad de este tipo de cursos, junto a la disponibilidad de acceso a la red que facilita una simple computadora o la banda ancha (McAuley, Stewart, Siemens \& Cormier 2010: 53). Mak, Williams y Mackness (2010) señalan que la preferencia por las herramientas participativas tiende a ser por los Foros, que permiten estrategias de demanda de respuesta rápida a cuestiones y dudas, en detrimento de Blogs, que sirven más para estrategias de reflexión y clarificación conceptual más "lentas".

\subsection{Perfil del usuario de los MOOC}

En segundo lugar hemos de tener en cuenta el sujeto que aprende, esto es, el perfil del aprendiz, el rol y las competencias que va a adquirir. En algunos estudios (Cita) se pone de manifiesto que el éxito de las plataformas MOOC, está condicionado al perfil de los usuarios, dado que no todos los usuarios consiguen terminar los cursos. El rol o el papel que juega el aprendiz a de ser activo y esto quiere decir que ha de participar en las actividades, aportando su propuesta en los foros, los chats, y demás recursos que ofrezca la plataforma con el fin de poder efectuar una construcción social del conocimiento y el aprendizaje en base a la colaboración entre los usuarios.

Un estudio de los usuarios de la plataforma MOOC realizado en 2013 por la Universidad de Stanford, apunta interesantes conclusiones sobre las diferencias de perfiles de estudiante que tienen más probabilidades de concluir con éxito este tipo de curso, y que en rasgos generales se corresponden con estudiantes de mayor nivel educativo, norteamericanos o de países con elevados niveles de desarrollo económico e industrial, incluso apuntando que los diseñadores deberían evitar el recurso excesivo a los vídeos explicativos produce una mayor dificultad para el seguimiento de los estudiantes sobre todo si son extranjeros o con bajo nivel educativo (Kizilcec; Piech \& Schneider, 2013). En el caso del estudio de Breslow et al. (2013) También resulta destacable la importante presencia de alumnos hispanohablantes (un 17\%, incluyendo Colombia con 6.00o y España con 5.000 alumnos como países cuarto y quinto respectivamente del ranking de naciones con mayor número de alumnos) por detrás de US (25.000), India (13.000) y Reino Unido (8.000).

En la misma línea, el estudio de la plataforma Canvas coincide al analizar el perfil de 1.834 estudiantes de la plataforma mediante la técnica de encuesta, en que se trata de un perfil altamente especializado con un porcentaje del $77 \%$ que disponen de titulación superior y un $42 \%$ educadores profesionales. Asimismo, coincide en que aquellos que consiguen superar satisfactoriamente este tipo de cursos son especialmente proclives a la utilización de los foros de participación e interacción de la comunidad 2.09. Un tercio de los alumnos consumen este tipo de productos de forma frecuente, lo que induce a pensar en una fidelización creciente del público objetivo de las plataformas MOOC.

9 Íbidem. 


\subsection{Modelo pedagógico}

El tercero de los elementos de análisis de las plataformas MOOC, se centra en el estudio de los modelos pedagógicos que las sustentan. Dado que este aspecto es muy extenso y que este propósito excede los objetivos del presente artículo, nos limitaremos a enumerar los tres modelosio más influyentes y que están en la base de la construcción de las plataformas MOOC: conductistas, sociocognitivos y del aprendizaje situado o situacional (Gros, 2002; García y Martín, 2002).

Las plataformas MOOC se fundamentan mayoritariamente en un modelo pedagógico sociocognitivo, donde los estudiantes construyen significados por sí mismos, como lo ponen de manifiesto la presencia de las variables que caracterizan este modelo teórico.

Sin embargo, hemos observado cómo las plataformas MOOC tienen elementos del diseño pedagógico asentado en los modelos conductistas, como lo demuestran el hecho de aquellas plataformas que contienen información para ser transmitida mediante el entrenamiento en las tareas a través de ejercicios, en donde el paso al siguiente nivel de dificultad viene determinado de antemano.

Otras propuestas metodológicas que encontramos en las plataformas MOOC se circunscriben dentro de los fundamentos teóricos del aprendizaje situado, en donde el contexto adquiere un papel esencial (Brown et al., 1989). Este enfoque es muy importante y se encuentra en la base de algunas de las propuestas metodológicas de las plataformas MOOC, en las que a partir del planteamiento de una situación compleja y problematizada y a través de la reflexión conjunta se van proponiendo nuevas tareas y retos a resolver por los participantes de la plataforma, de modo que se simulan o representan figuradamente los supuestos aplicables a un entorno real.

\subsection{Modelo de desarrollo software}

Nuestro análisis muestra una tendencia hacia las plataformas de código propietario que pretenden desarrollar un modelo de negocio en base a comunidades de educadores ligados a las mismas, con diferentes estrategias de retorno económico como son el pago por certificados o reconocimiento académico en forma de créditos, o bien mediante suscripción de usuarios o fórmulas publicitarias. La alternativa principal es la de las plataformas de código abierto, que respetan más exactamente los requisitos del concepto MOOC. En este sentido dos son los entornos de desarrollo software principales.

La liberación como código fuente abierto de edX en junio de 2013, unido al prestigio del consorcio que lo promueve, le convierte en el software de mayor proyección en el ámbito de desarrollo de plataformas públicas y abiertas, junto al software OpenMooc que adoptó con antelación dicha estrategia de apertura. edX ha sido liberado bajo una licencia de software AGPLv3 mientras que OpenMOOC recurre a una más permisiva licencia Apache v2.

10 Está síntesis es la que también utilizan otros autores como Freitas y Jarvis (2007) para evaluar otras herramientas 3.o. de aprendizaje. 


\section{Conclusiones}

Los datos indican elementos de debilidad en la retención de estudiantes por parte de las plataformas MOOC, así como también apuntan a desigualdades relativas al acceso a estas plataformas de e-learning. Asimismo apuntan a graves carencias desde el punto de vista de la estrategia de diseño de las plataformas. Dado el elemento crítico que supone la capacidad de interacción asociada a los cursos MOOC, podemos considerar necesario atender al análisis de los modelos pedagógicos y sus requerimientos antes de la construcción de una plataforma MOOC, de modo que aseguremos que existe una congruencia entre la concepción del aprendizaje en una plataforma MOOC, y el modelo o modelos pedagógicos que la fundamentan a priori. Resulta llamativo que estudios como el de Khalil y Ebner (2013) apunten a que la demanda de más interacción por parte de los estudiantes de los MOOC hacia los instructores sea un elemento de insatisfacción. ¿Estamos asistiendo al inicio de la curva descendente del conocido hype-cycle de Gartner respecto a esta tecnología e-learning que tantas expectativas ha generado en la sociedad de la información (Fenn \& Time, 2007)? ¿Es posible que el talón de Aquiles de los MOOC sea la ausencia de interacción con el educador?

En este sentido, tomando como referencia el modelo de interactividad en e-learning desarrollado por Salmon (2002) podríamos considerar que la capacidad de motivación y dinamización de la participación por el instructor o moderador de las herramientas 2.o resulte en algún modo esencial para conseguir avanzar en los pasos deseados de alcanzar un aprendizaje online activo (Salmon, 2002; Chen \& Chen, 2007), por lo que sería interesante comparar las Plataformas desde el punto de vista de que dispongan de sistemas de motivación e interacción entre los instructores y los alumnos.

En consecuencia, una de las condiciones a las que se debe prestar mayor atención, en cuanto al diseño pedagógico de las plataformas MOOC, es a la estrategia de implicación activa del sujeto, siendo esto una de las tareas prioritarias en la actualidad para estos entornos Web (Norvig, 2012).

Nuestro trabajo en síntesis pretende arrojar tres vías de reflexión sobre los MOOC :

- La necesidad de determinar si el objetivo es la adquisición de competencias o la necesidad de tener una formación certificada. Pensaríamos que los dos aspectos no son excluyente sobre todo en la formación durante toda la vida (lifelong learning) como existe en la Unión Europea.

- La urgencia de establecer un pliego de condiciones o un libro blanco sobre los elementos esenciales que deben reunir los MOOC. Para ello sería elemento indispensable la creación de comités que integren empresarios, representantes del estado y del mundo universitario en su definición.

- El equilibrio entre los diseños pedagógicos, las plataformas y la adaptación de herramientas interactivas en los entornos Web. Solamente esta ecuación podrá responder a una verdadera innovación pedagógica, como resulta por ejemplo el caso de los juegos serios (serious games) (Caron, \& Heutte, 2013). 


\section{Referencias bibliográficas.}

Avila, P. \& Bosco, D. (2001). Ambientes Virtuales de Aprendizaje. Una nueva experiencia. Recuperado el 29 de julio de 2013 de http://investigacion.ilce.edu.mx/panel_control/doc/c37ambientes.pdf

Breslow, L.; Pritchard, D. E.; DeBoer, J.; Stump, G. S.; Ho, A. D. and Seaton, T. D. (2013). Studying Learning in the Worldwide Classroom: Research into edX's First MOOC. Research \& Practices in Assessment, vol. 8, 13-25. Recuperado el 7 de junio de 2013 de http://www.rpajournal.com/dev/wpcontent/uploads/2013/05/SF2.pdf

Brown, J. S., Collins, A. \& Duguid, P. (1989). Situated cognition and the culture of learning. Educational Researcher, 18, 32-42.

Brown, J. S., \& Adler, R. P. (2008). Minds on fire: Open education, the long tail, and learning 2.o. Educause Review, vol. 43, n. 1, pp. 1632, Recuperado el 3 de Julio de 2013

de http://www.educause.edu/EDUCAUSE+Review/EDUCAUSEReviewMagazineVol ume43/MindsonFireOpenEducationtheLon/162420

Caron, P-A, \& Heutte, J. (2013). MOOC et gamification: vers une ingénierie de formation a utotélique? Environnements Informatiques pour l'Apprentissage Humain. Conférence EIAH'2013, Toulouse. Recuperado el 17 de junio de 2013 de http://ateliermooceiah2013.files.wordpress.com/2013/o5/caron.pdf

Chen, Y.-J. \& Chen, P.-C. (2007). Effects of Online Interaction on Adult Students' Satisfaction and Learning. The Journal of Human Resource and Adult Learning, Vol. 3, Num. 2, 78-89

Cross, S. (2013). Evaluation of the OLDS MOOC curriculum design course: participant perspectives, expectations and experiences. OLDS MOOC Project, Milton Keynes. The Open Uiversity en http://oro.open.ac.uk/37836/1/EvaluationReport_OLDSMOOC_v1.o.pdf] (25 de Julio de 2013).

Derycke, A. (2013). Pour une analyse critique des MOOC à la lumière de la nouvelle économie. Atelier MOOC, EIAH, Toulouse. Recuperado el 14 de junio de 2013 de http://ateliermooceiah2013.files.wordpress.com/2013/05/derycke.pdf

Dziorny, M. (2007). Digital Game-based Learning and dyslexia in higher education. In R. Carlsen et al. (Eds.), Proceedings of Society for Information Technology \& Teacher Education International Conference 2007 (pp. 1189-1197). Chesapeake, VA: AACE. Recuperado el 23 de Julio de 2013 de http://www.editlib.org/p/24720

Esposito, A. (2012). Research ethics in emerging forms of online learning: issues arising from a hypothetical study on a MOOC. The Electronic Journal of eLearning, 10 (3), 315-325

Fenn, J., \& Time, M. (2007). Understanding Gartner's Hype Cycles, 2007. 
Freitas, S., \& Jarvis, S. (2007). Serious games engaging training solutions: A research and development project for supporting training needs. British Journal of Educational Technology, 38 (3), 523-525

García, A. \& Martín, A. V. (2002). Caracterización pedagógica de los entornos virtuales de aprendizaje. Teoría de la Educación, 14, 67-92.

Gros, B. (2002). Burrhus Frederic Skinner y la tecnología en la enseñanza. En J. Trilla, (coord.), El legado pedagógico del siglo XX para la escuela del siglo XXI (pp 229248). Barcelona: Graó

Khalil, H. \& Ebner, M. (2013). How satisfied are you with your MOOC? - A Research Study on Interaction in Huge Online Courses. En J. Herrington et al. (Eds.), Proceedings of World Conference on Educational Multimedia, Hypermedia and Telecommunications (pp. 830-839). Chesapeake, VA: AACE.

Kizilcec, R. F.; Piech, C. \& Schneider, E. (2013). Deconstructing Disengagement: Analyzing Learner Subpopulations in Massive Open Online Courses. Stanford University Paper Series. Recuperado el 18 de Junio de 2013 de http://www.stanford.edu/ cpiech/bio/papers/deconstructingDisengagement.pdf

Kohler, F., Jay, N., Ducreau, F., Casanova, G., Kohler, Ch., \& Benhamou, A.C. (2013). COURLIS (COURs en LIgne de Statistiques appliquées) Un MOOC francophone innovant. Recuperado el 30 de Julio de 2013 de http://documents.irevues.inist.fr/bitstream/handle/2042/49205/HEGEL_2013_ 3_1-27.pdf?sequence $=1$

Kop R., Hill A. (2008). Connectivism: Learning theory of the future or vestige of the past? The International Review of Research in Open and Distance Learning, Vol.9 $n^{\circ} 3$, ISSN: 1492-3831. Recuperado el 13 de junio de 2013 de http://www.irrodl.org/index.php/irrodl/article/view/523/1103

Kop, R. (2011). The challenges to connectivist learning on open online networks: Learning experiences during a massive open online course", IRRODL, vol. 12, n.3,

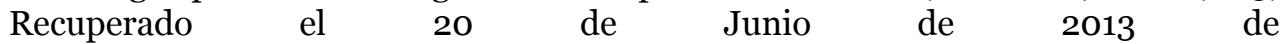
http://www.irrodl.org/index.php/irrodl/article/view/882

Krichen, J.P. (2007). Investigating learning styles in the online educational environment. Paper presented at the Conference on Information Techonology Education (CITC). In Sweeney, B., Feintein, D.L. \& Ekstrom, J.J. (Eds.) Proceedings of the 8th ACM SIGITE conference on Information technology education (pp.127-134). ACM: Association for Computing Machonery.

Mak, S., Williams, Roy \& Mackness, J. (2010). Blogs and forums as communication and learning tools in a MOOC. In: Proceedings of the 7 th International Conference on Networked Learning 2010. University of Lancaster, Lancaster, pp. 275-285.

McAuley, A.; Stewart, B.; Siemens, G. \& Cormier, D. (2010). The MOOC Model for Digital Practice: Massive Open Online Courses. Digital ways of knowing and learning. University of Prince Edward Island. Recuperado el 6 de Julio de 2013 https://oerknowledgecloud.org/sites/oerknowledgecloud.org/files/MOOC_Final_ o.pdf 
McLellan, H. (1997). Creating Virtual Communities via the Web. En B. Khan Ed. WebBased Instruction. New Jersey: Englewood Cliffs, 185-190.

Norvig, P. (2012). Peter Norvig: The 100,000-student classroom, Recuperado el 19 de Junio de 2013 de http://www.ted.com/talks/peter_norvig_the_100_ooo_student_classroom.html

Parr, Chris (2013, May 10). Not Staying the Course. Times Higher Education. $\begin{array}{llllll}\text { Recuperado el } & 20 & \text { de } & \text { Julio } & \text { de } & 2013\end{array}$ http://www.insidehighered.com/news/2013/05/10/new-study-low-Mooccompletion-rates

Prensky, M. (2010). Teaching Digital Natives. Thousand Oaks (Cal.): Corwin Press

Salmon, G. (2002). E-tivities: The key to active online learning. London: Kogan Page Ltd.

Teplechuk, Evgenia (2013). Emergent models of Massive Open Online Courses: an exploration of sustainable practices for MOOC institutions in the context of the launch of MOOCs at the University of Edinburgh. Dissertation Presented for the Degree of MBA. University of Edinburgh. Recuperado el 3 de Mayo de 2013 de http://www.era.lib.ed.ac.uk/bitstream/1842/7536/1/MOOCs_MBADissertationTe plechuk_Master.pdf 